\title{
Stellar discs in massive galaxies
}

\author{
D. Krajnović, ${ }^{1}$ K. Alatalo, ${ }^{2}$ L. Blitz, ${ }^{2}$ M. Bois,${ }^{3}$ F. Bournaud, ${ }^{4}$
} M. Bureau, ${ }^{5}$ M. Cappellari, ${ }^{5}$ R. L. Davies,${ }^{5}$ T. A. Davis,${ }^{1}$

P. T. de Zeeuw,${ }^{1,6}$ E. Emsellem, ${ }^{1,8}$ S. Khochfar, ${ }^{7}$ H. Kuntschner, ${ }^{1}$ R. M. McDermid, ${ }^{9}$ R. Morganti, ${ }^{10}$ T. Naab,${ }^{11}$ M. Sarzi,${ }^{12}$ N. Scott,${ }^{13}$ P. Serra, ${ }^{10}$ A. Weijmans ${ }^{14}$ and L. M. Young ${ }^{15}$

${ }^{1}$ ESO, Garching, Germany; ${ }^{2}$ University of California, Berkeley, USA; ${ }^{3}$ Observatoire de Paris, France; ${ }^{4}$ Université Paris Diderot, France; ${ }^{5}$ University of Oxford, UK; ${ }^{6}$ Leiden University, The Netherlands; ${ }^{7} \mathrm{MPE}$, Garching, Germany; ${ }^{8}$ Université de Lyon, France; ${ }^{9}$ Gemini Observatory,

Hilo, USA; ${ }^{10}$ ASTRON, Dwingeloo, The Netherlands; ${ }^{11}$ MPA, Garching, Germany;

${ }^{12}$ University of Hertfordshire, Hatfield, UK; ${ }^{13}$ CAS, Swinburne University of Technology, Australia; ${ }^{14}$ University of Toronto Canada; ${ }^{15}$ New Mexico Tech, Socorro, USA

Summary. Excluding those unsettled systems undergoing mergers, bright galaxies come in two flavours: with and without discs. In this work we look for photometric evidence for presence of discs and compare it with kinematic results of the ATLAS ${ }^{3 \mathrm{D}}$ survey (Cappellari et al. 2011). We fit a Sérsic (1968) function to azimuthally averaged light profiles of ATLAS $^{3 \mathrm{D}}$ galaxies to derive single component fits and, subsequently, we fit a combination of the Sérsic function (free index $n$ ) and an exponential function $(n=1)$ with the purpose of decomposing the light profiles into "bulge" and "disc" components (B+D model) of all non-barred sample galaxies. We compare the residuals of the $\mathrm{B}+\mathrm{D}$ models with those of the single Sérsic fits and select the B+D model as preferred only when the improvement is substantial and there are no correlations within residuals. We find that the high angular momentum objects (fast rotators) are disc dominated systems with bulges of typically low $n$ (when their light profiles can be decomposed) or are best represented with a single Sérsic function with a low Sérsic index $(n<3)$. Single component systems with large Sérsic indices are characteristic of low angular momentum objects (slow rotators).
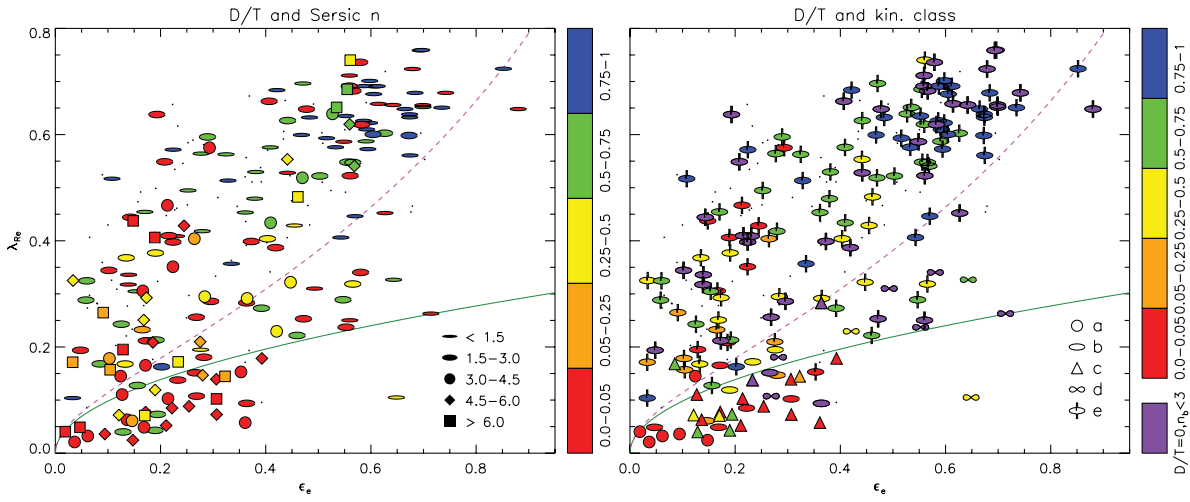

Figure 1. Angular momentum $\lambda_{R}$ versus ellipticity $\epsilon$ of ATLAS ${ }^{3 \mathrm{D}}$ galaxies. Left: Symbols represent Sérsic indices as shown on the legend. Right: Symbols show different types of kinematics from Krajnović et al. (2011) and are described in the legend: $a$ - non rotating galaxies, $b$ featureless non-regular rotators, $c$ - KDC, $d$ - counter-rotating discs, and $e$ - regular rotators. On both plots colours quantify $\mathrm{D} / \mathrm{T}$ ratios, as shown on the respective colour bars.

\section{References}

Cappellari, M., et al. 2011,MNRAS,413,813

Sérsic, J. L.,1968, Atlas de galaxias australes, Observatorio Astrónomico, Córdoba, Argentina 